\title{
Statistical comments on "Combined effect of whole-body vibration and ambient lighting on human discomfort, heart rate, and reaction time"
}

\author{
Afshin Amirpour ${ }^{1} \cdot$ Reihaneh Zavar $^{1} \cdot$ Milad Ebrahimi $^{2} \mathbb{D}$
}

Received: 4 April 2019 / Accepted: 6 September 2019 / Published online: 16 September 2019

(c) Springer-Verlag GmbH Germany, part of Springer Nature 2019

\section{Dear Editor}

With great interest and attention, we read a recent paper by Monnazam et al. (2018) entitled "Combined effect of wholebody vibration and ambient lighting on human discomfort, heart rate, and reaction time". In this study, the authors have been studied the effect of whole-body vibration and ambient lighting, as well as their combined effect on human discomfort, heart rate, and reaction time in laboratory conditions. They evaluated discomfort rate, heart rate and reaction time in different light intensities and vibration accelerations in 44 men. Since they assessed the discomfort rate, heart rate and reaction time values in the same subjects in four different vibration accelerations and three different light intensities, so their measurements are completely dependent. As stated in the statistical analysis section and the Tables 2 and 4 of the article, the authors used one-way analysis of variance (ANOVA) followed by Scheffe post hoc test and Kruskal-Wallis test to compare the discomfort rate, heart rate and reaction time between different light intensities and vibration accelerations. They found significant difference in discomfort rate and heart rate between different vibration accelerations $(P<0.001)$ and also significant difference between different light intensities regarding discomfort rate $(P<0.001)$. One way ANOVA and Kruskal-Wallis tests are used to determine statistically significant differences of parametric and non-parametric continuous variable between two or more independent groups, respectively (Gaddis 1998). Therefore, after investigation the normality of numerical

Milad Ebrahimi

milad.labsc@yahoo.com

1 Cardiac Rehabilitation Research Center, Cardiovascular Research Institute, Isfahan University of Medical Sciences, Isfahan, Iran

2 Department of Immunology, School of Medicine, Shahed University, 802016 Tehran, Iran data, the authors must use repeated measures ANOVA and Friedman tests to compare the means of discomfort rate, heart rate and reaction time between four different vibration accelerations and three different light intensities. They also must use paired $t$ test or Wilcoxon signed-rank test to compare the means of discomfort rate, heart rate and reaction time between each two different vibration accelerations and light intensities.

Taken to gather, analysis of differences of discomfort rate, heart rate and reaction time between four different vibration accelerations and three different light intensities with appropriate statistical tests is strongly suggest to improve the reliability of results of this valuable study.

\section{Compliance with ethical standards}

Conflict of interest The authors report no conflict of interest.

\section{References}

Gaddis ML (1998) Statistical methodology: IV. Analysis of variance, analysis of co variance, and multivariate analysis of variance. Acad Emerg Med 5(3):258-265

Monazzam MR et al (2018) Combined effect of whole-body vibration and ambient lighting on human discomfort, heart rate, and reaction time. Int Arch Occup Environ Health 91(5):537-545

Publisher's Note Springer Nature remains neutral with regard to jurisdictional claims in published maps and institutional affiliations. 